\title{
CARACTERIZACION ESTRUCTURAL DE LA MADERA DE Pinus radiata D. Don DEL PAIS VASCO (ESPAÑA) ACORDE A LAS MODIFICACIONES NORMATIVAS
}

\section{STRUCTURAL CHARACTERIZATION OF Pinus radiata D. Don TIMBER FROM PAIS VASCO (SPAIN) ACCORDING TO STANDARD MODIFICATIONS}

\author{
Hermoso E. ${ }^{1}$, Carballo J. ${ }^{2}$, Fernández-Golfín J.I. ${ }^{3}$
}

\section{RESUMEN}

En las últimas décadas en Europa se ha promovido la promulgación de Códigos de Práctica, Directivas y Normas Armonizadas de obligado cumplimiento para los países miembros, que afectan a la madera en la construcción y que son imprescindibles para favorecer al material en su uso estructural. Debido al carácter dinámico intrínseco a las normas, y necesario para su funcionalidad, éstas sufren modificaciones en el tiempo por lo que es necesario confrontarlas con el material realmente existente. En este trabajo se realizó un muestreo representativo en el País Vasco sobre una de las especies de madera más habituales en la construcción en España como es el pino radiata (Pinus radiata D.Don), buscando la veracidad de lo disponible en el mercado tanto en calidades como en dimensiones, y se aplicó la metodología normalizada y estipulada para su caracterización. Además, en cada una de las piezas se aplicaron los criterios de clasificación visual establecidos en la norma española UNE 56.544 para la estimación de calidades. En los resultados se presentan las conclusiones obtenidas tras la aplicación de las últimas variaciones de la normativa para la determinación de las propiedades mecánicas, las cuales están implicadas en los análisis de la caracterización estructural de la madera y condicionan la asignación resistente final.

Palabras clave: Pinus radiata, madera estructural, clases resistentes.

\begin{abstract}
In the last decades in Europe the promulgation of Practice Codes, Directives and Harmonised Standards of obliged fulfilment for the member countries was promoted. They affect to wood in construction and are essential to improve the material in the structural use. Due to the dynamic character proper of standards, and necessary for their functionality, these suffer modifications in the time that are necessary to check in the really existing material. In this work, a representative sampling on one of the usual species of wood in the construction in Spain, as it is the radiata pine, was carried out, looking for the veracity of the available material in the market both in qualities and sections. The standard methodology stipulated for the characterization was applied. Additionally, in each piece, the visual
\end{abstract}

\footnotetext{
${ }^{1}$ Investigador y Director Técnico del Laboratorio de Estructuras de Madera del INIA. Instituto Nacional de Investigación y Tecnología Agrario-Alimentaria. INIA. 28040 Madrid, España.

${ }^{2}$ Técnico del Laboratorio de Estructuras de Madera del INIA.

${ }^{3}$ Investigador y Director del Laboratorio de Estructuras de Madera del INIA.

Autor para correspondencia: hermoso@inia.es

Recibido: 18.07.2007. Aceptado: 20.09.2007
} 
classification criteria established in the Spanish standard UNE 56.544 for the estimation of the strength grades, was applied. The results set forth the conclusions obtained after the application of the last changes of the regulation for the determination of mechanical properties, which are involved in the analyses of the structural characterization of timber and determine the final strength assignation.

Keywords: Pinus radiata, structural timber, strength classes.

\section{INTRODUCCION}

Según datos del segundo Inventario Forestal Nacional, del Ministerio de Medio Ambiente (el tercer Inventario Forestal Nacional de España estará terminado en el año 2007), la superficie forestal española es de aproximadamente 26 millones de ha, lo que supone el $51 \%$ del territorio, de las cuales 13.905 .000 son arboladas. La mayor parte se encuentran en las provincias que conforman la zona Atlántica, con una extensión de casi el $34 \%$ respecto al total arbolado. El área ocupada por bosques de coníferas es 5,7 millones de ha, siendo la distribución para las especies principales la siguiente:

- Pinus pinaster Ait.: 1.134 .000 ha

- Pinus sylvestris L.: 1.088 .000 ha

- Pinus nigra Arn.: 586.000 ha

- Pinus radiata D. Don.: 199.000 ha

La especie objeto de este trabajo, el pino radiata (Pinus radiata D. Don.), es la conífera más utilizada en plantaciones forestales en España y está repartida por el País Vasco en su mayoría (137.500 ha), Galicia y toda la cornisa cantábrica, zonas donde se dan las condiciones necesarias para su crecimiento: más de $900 \mathrm{~mm}$ anuales de precipitación y temperaturas suaves, no sobrepasando las plantaciones los $500 \mathrm{~m}$ de altitud (Ceballos y Ruiz de la Torre, 1979).

Aún cuando desde el punto de vista de superficie esta madera tan sólo representa el 3,6\% del total ocupado por coníferas en España, su producción supone el $25 \%$ del global. Con un turno de 35 años genera $23,7 \mathrm{~m}^{3} / \mathrm{ha} /$ año.

Esta situación se ha traducido en la creación, concretamente en el País Vasco, de un fuerte tejido industrial basado en la transformación de su madera y en particular, está teniendo una buena aceptación su uso como material estructural.

Históricamente las masas de pino radiata han estado sujetas a una selvicultura intensiva, por lo que su madera ha sido utilizada de forma tradicional para proveer de astillas a las industrias del papel así como de materia prima para la industria de envases. Sin embargo, la forma del fuste del pino radiata, sin curvaturas, permite la obtención de longitudes de troza que sitúan a su madera en una posición muy ventajosa para la utilización en la construcción.

El estudio sobre esta especie también resulta trascendente para el sector forestal ya que las posibilidades de esta madera en la construcción se adaptan a las calidades medias de las producciones españolas (Fernández-Golfín y Díez, 1999), generando además un precio de venta medio sensiblemente más alto que en los sectores tradicionales a los que se destina. La consolidación de este mercado de la madera estructural de pino radiata en España permitirá la mejora de la rentabilidad económica de los aserraderos así como, por extensión, del sector de los propietarios de montes, que podrían obtener mayores ingresos por su materia prima. Debe destacarse que una mejora en la rentabilidad de los montes debe redundar en su limpieza y en la posibilidad de realizar en ellos prácticas selvícolas, hoy no habituales en España, como las podas, lo que a su vez tendría un efecto positivo sobre la calidad media de la materia prima producida. 
El primer paso debe ir dirigido a contribuir al conocimiento de sus propiedades estructurales para el uso adecuado en el mercado de la construcción. Recientemente han sido creadas diversas normativas tanto a nivel europeo como a nivel nacional que establecen el ámbito que regula este proceso, demandando el esfuerzo de actualizar las metodologías y datos de las especies madereras habituales conforme a los nuevos requisitos.

A comienzos de los 90 se realizaron estudios en el INIA sobre la madera de pino radiata ya orientados a la caracterización de sus propiedades estructurales (Ortiz et al., 1990; López de Roma et al.,1991; Ortiz y Martínez, 1991). Por diversas razones, entonces sólo se incluyeron dos secciones de ensayo (40x100 mm y 50x150 mm), lo que incumple las especificaciones de la actual norma UNE-EN 384, que obliga a considerar, por motivos de seguridad en los resultados, las secciones representativas del rango comercializado. Para generar un lenguaje común en el tratamiento de la madera de uso estructural, se ha definido en la Unión Europea un sistema denominado de Clases resistentes, recogido en la norma UNE-EN 338, que agrupa los valores de las propiedades necesarias para los cálculos. Pues bien, la introducción de los resultados de la asignación a clases resistentes derivados de la caracterización de los cuatro pinos más utilizados en la construcción en España, en la norma UNE-EN 1912 donde se relaciona la clase resistente según calidades visuales y procedencia de la especie, y de referencia para cualquier prescriptor, es otro motivo que hace necesaria la ejecución de este trabajo.

Además, la particularidad del rápido crecimiento de esta especie invita a comprobar si condiciona sus propiedades elasto-mecánicas a lo largo del tiempo.

El estudio se ha realizado en el Laboratorio de Estructuras de Madera del INIA, el cual desde mediados de los años 80, viene trabajando en la caracterización de las principales maderas españolas de susceptible uso estructural. Su experiencia ha sido adquirida a través de la caracterización estructural de diversas maderas de procedencia española como Pinus pinaster (Ortiz et al., 1990), Pinus radiata (Ortiz y Martínez, 1991; López de Roma et al., 1991), así como sobre Pinus sylvestris (FernándezGolfín et al., 1997; Hermoso, 2001; Fernández-Golfín et al., 2002; Hermoso et al., 2002, 2003) y Pinus nigra (Fernández-Golfín et al., 2000; 2001 a, b y c; Fernández-Golfín et al., 2003; Conde, 2004).

\section{OBJETIVOS}

Como principal objetivo se plantea la obtención de los resultados de la caracterización mecánica de la madera de pino radiata del norte de España acorde a las exigencias metodológicas que la normativa europea establece al efecto, lo que incluye aportar los valores de sus propiedades elasto-mecánicas como madera de uso estructural y establecer las relaciones que las ligan entre sí.

\section{MATERIALES Y MÉTODOS}

En la elección de la muestra se tuvieron presentes los requisitos novedosos que la norma UNE-EN 384 establece respecto a la edición anterior para la caracterización de una especie de madera en el seno de la Unión Europea. Principalmente estuvieron relacionados con la representatividad de las secciones presentes en el mercado en la población de estudio. La propia normativa establece una penalización sobre los resultados cuando el número de muestras no es acorde a lo mínimo estipulado. El estudio se ha realizado sobre un total de 768 vigas de pino radiata procedentes del País Vasco (Norte de España) y para mantener la representatividad de la zona y recoger su variabilidad, las muestras se reúnen de varios aserraderos cuyo material proviene de material señalado para corta en distintos montes de la región. De esta forma se asegura realizar el análisis sobre el material realmente comercializado. Para cumplir el objetivo del trabajo, las secciones analizadas fueron ampliadas respecto a los estudios 
anteriores, teniendo finalmente escuadrías de $100 \times 50 \mathrm{~mm}, 150 \times 40 \mathrm{~mm}, 150 \times 50 \mathrm{~mm}, 150 \times 70 \mathrm{~mm}$, $200 \times 50 \mathrm{~mm}$ y $200 \times 70 \mathrm{~mm}$, con longitudes variables desde 2 hasta 4 metros. La muestra de 150x50 mm fue la seleccionada para realizar un muestreo más intensivo sobre los aserraderos por ser la utilizada tradicionalmente de referencia; por esta razón su número es sensiblemente mayor a las otras dimensiones.

Las piezas se secaron a la humedad determinada por la normativa para su evaluación, alrededor del $12 \%$. Previamente a cualquier ensayo se rechazaron las piezas deformadas durante el secado; sobre el resto se aplicó la clasificación visual según la norma española UNE 56.544 que aporta los criterios para determinar distintas calidades resistentes de la madera de pinos españoles. Estas calidades se denominan ME1 (Madera Estructural de primera) y ME2 (Madera Estructural de segunda). Las piezas que no se encuadran dentro de éstas, son piezas de rechazo no aptas para el uso estructural.

Posteriormente se realizaron varios ensayos basados en técnicas no destructivas para la evaluación de su resistencia, que no son objeto de esta exposición, y para la determinación del punto más débil necesario para los análisis posteriores.

La clasificación de la madera estructural en un lenguaje común en la norma UNE-EN 338 queda definida mediante clases resistentes designadas por la letra $C$ al inicio, correspondiente a coníferas, seguidas de un número relativo al valor de la resistencia a la flexión (ej: C18). Para la asignación de clases resistentes a calidades visuales es suficiente el cálculo de tres propiedades: el módulo de elasticidad $(E)$, el módulo de rotura $(f)$ y la densidad $(\rho)$. De ellas se puede obtener el resto mediante fórmulas definidas en la norma UNE-EN 384. Por esta razón se las denomina propiedades clasificadoras y se efectuaron ensayos sólo para determinación de estas tres.

El procedimiento para la obtención de las propiedades elasto-mecánicas de la madera se realizó acorde a la norma de ensayos UNE-EN 408, la cual define la disposición de la viga a flexión biapoyada y la aplicación de dos cargas en el tercio central (Figura 1). Manteniendo la carga en el tramo elástico se obtiene el módulo de elasticidad medido en el lado traccionado de la pieza $\left(E_{m g}\right)$, aunque la norma también permite su cálculo en la línea neutra o de forma localizada en el tercio central donde se produce el momento máximo. Por facilidad de ejecución se eligió el primer tipo. La pieza se sitúa con el punto más débil en el tercio central, lo que supone una medida adicional de seguridad en los resultados. Finalmente se continúa la carga de la viga en flexión hasta la rotura para conocer su valor real de resistencia que sirva para evaluar la calidad de sus predictores.

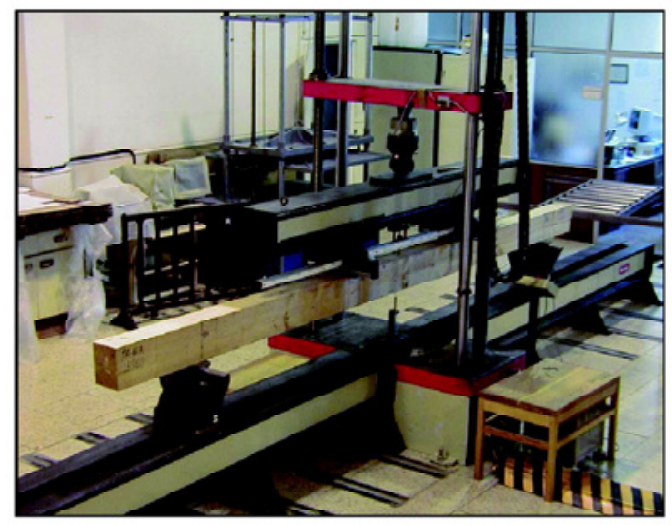

Figura 1. Configuración del ensayo a flexión según norma UNE-EN 408. 
La zona de rotura se clasifica nuevamente según los criterios visuales y se guarda un dibujo por si en el futuro fuera necesario una reevaluación debido a la modificación de la normativa. Asimismo, sirve para determinar el valor relativo del nudo respecto a la cara (DCAREL) y el canto (DCTOREL) y analizar posteriormente su efecto sobre la calidad de la madera. Para el cálculo de la densidad se extrae una probeta de la sección intacta y sin defectos cercana a la zona de rotura.

Los valores de las propiedades se obtuvieron según la norma UNE-EN 384 en donde además se definen las correcciones a aplicar sobre el módulo de elasticidad y la densidad en caso de que la humedad de la pieza difiera del $12 \%$ de referencia. Igualmente, para poder comparar los resultados de la resistencia a la flexión de las distintas secciones, fija una altura de cara de referencia de $150 \mathrm{~mm}$ estableciéndose correcciones para los casos que varíe.

Debido a que el módulo de elasticidad puede ser calculado de diversas formas, el valor a introducir para conocer la clase resistente resultante debe ser $E_{m l}$ (UNE-EN 384), valor libre de la influencia de cortantes, calculado como resultado de la aplicación de la siguiente fórmula:

$$
E_{m l}=1,3 \times E_{m g}-2690
$$

siendo $E_{m g}$ el módulo de elasticidad calculado de forma global. Igualmente debe introducirse el valor de la resistencia correspondiente al quinto percentil y el valor de la densidad característico, calculado a partir de la densidad media $\left(\rho_{m}\right)$ y de la desviación típica $(\sigma)$, según:

$$
\rho_{05}=\rho_{m}-1,654 \times \sigma
$$

Los datos se analizaron utilizando la estadística clásica del programa Statgraphics Centurión v.15.2. También es de destacar en este apartado que de la ejecución se guarda una muestra de cada pieza (la empleada para el cálculo de la densidad) de forma que en el día de mañana se pueda llevar a cabo con ellas estudios de genómica y proteómica que permitan analizar si determinadas variables que afectan a la calidad de la madera son heredables o no y, por tanto, susceptibles de mejora genética.

\section{RESULTADOS Y DISCUSION}

La descripción detallada de la muestra de ensayo según las secciones y el número de piezas estudiadas, se presenta en la Tabla 1. La norma UNE-EN 384, establece que la muestra utilizada para la caracterización de las propiedades de una madera, debe ser representativa de los tamaños y calidades presentes en la producción y además, cada una debe incorporar al menos 40 piezas, ya que en caso contrario, dispone la aplicación de penalizaciones. Debido a que existen dos calidades según la norma de clasificación UNE 56.544, la muestra elegida para el estudio incorpora el número suficiente de piezas para asegurar al menos esa cantidad en cada una de ellas.

Tabla 1. Descripción de la muestra de estudio.

\begin{tabular}{|c|c|}
\hline $\begin{array}{c}\text { SECCIÓN } \\
(\mathbf{m m})\end{array}$ & $\mathbf{N}^{\mathbf{0}}$ de piezas \\
\hline $100 \times 50$ & 84 \\
\hline $150 \times 40$ & 89 \\
\hline $150 \times 50$ & 331 \\
\hline $150 \times 70$ & 83 \\
\hline $200 \times 50$ & 93 \\
\hline $200 \times 70$ & 86 \\
\hline
\end{tabular}


La asignación de clases resistentes se efectúa para calidades visuales, por lo que el estudio inicial debe ir encaminado a comprobar si la metodología de clasificación aplicada sobre esta especie consigue la esperada diferenciación entre resistencias. Mediante análisis de varianza se analizó si las distintas clases visuales poseían propiedades mecánicas significativamente distintas o no. En la Figura 2 se aprecia dicho estudio para la resistencia a la flexión.

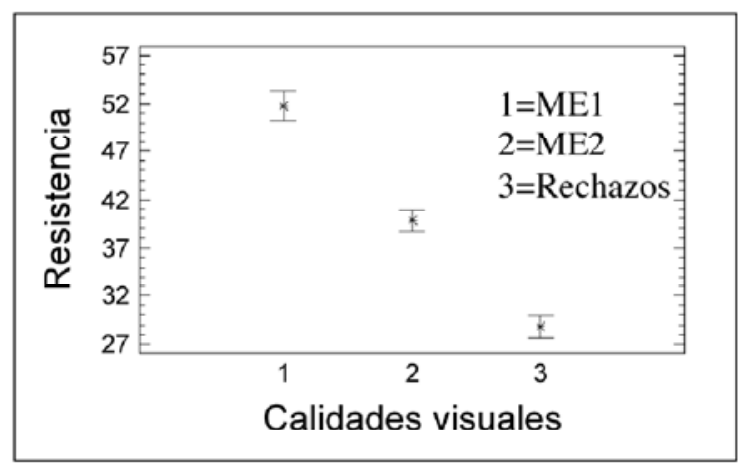

Figura 2. Análisis ANOVA calidad visual-resistencia a la flexión $\left(\mathrm{N} / \mathrm{mm}^{2}\right)$

En la Tabla 2 se presentan los datos relativos a la resistencia, rigidez y densidad media para las diferentes secciones, resultantes de los ensayos según UNE-EN 408. Es destacable la uniformidad de los valores obtenidos en todas ellas salvo para la sección de 150x50 mm, superior a la media de otras secciones. La explicación puede deberse a que estas piezas se obtienen de la zona del tronco de mejor calidad, ya que las piezas de menor sección se suelen extraer de las trozas superiores y las mayores de las inferiores, incluyendo singularidades como la médula y la madera juvenil en gran número de ellas, que afectan a sus propiedades.

Tabla 2. Valores medios de las propiedades elasto-mecánicas en función de la sección.

\begin{tabular}{|c|c|c|c|}
\hline & $\begin{array}{c}\text { Resistencia } \\
\mathrm{N} / \mathbf{m m}^{2}\end{array}$ & $\begin{array}{l}\text { Rigidez } \\
\text { N/mm }\end{array}$ & \multirow{2}{*}{$\begin{array}{c}\begin{array}{c}\text { Densidad } \\
\mathrm{kg} / \mathbf{m}^{3}\end{array} \\
\rho_{m} \\
\end{array}$} \\
\hline & $f_{m}$ & $\boldsymbol{E}_{m g}$ & \\
\hline $\begin{array}{c}\text { SECCIÓN } \\
\text { (mm) } \\
100 \times 50\end{array}$ & 30,5 & 8230 & 468 \\
\hline $\begin{array}{l}100 \times 50 \\
150 \times 40\end{array}$ & 32,7 & 8438 & 468 \\
\hline \multirow{2}{*}{\begin{tabular}{|l|}
$150 \times 50$ \\
$150 \times 70$ \\
\end{tabular}} & 45,5 & 10796 & 511 \\
\hline & 32,5 & 8619 & 466 \\
\hline \multirow{2}{*}{$\begin{array}{l}200 \times 50 \\
200 \times 70\end{array}$} & 33,1 & 9496 & 473 \\
\hline & 33,3 & 8778 & 453 \\
\hline
\end{tabular}

Los resultados de la asignación de clases resistentes a clases de calidad visual se indican en la Tabla 3. Pese a las dudas que pudieran surgir debido al tipo de manejo selvícola aplicado sobre esta especie orientado a turnos cortos y a crecimientos rápidos, se observa que la calidad de la madera mantiene las asignaciones resistentes esperadas en la línea de los datos anteriores $(\mathrm{ME} 1=\mathrm{C} 24$ y ME2 = C18) (UNE-EN 338; Fernández-Golfín y Díez, 1999).

Los rendimientos sin embargo no siguen la tendencia de otros pinos españoles ( $25 \%$ - $50 \%$ - $25 \%$ ), ya que se detecta una disminución de las piezas clasificadas como segundas a favor de las piezas 
rechazadas. Esto puede deberse a que los requisitos de la norma de clasificación visual no sean los más apropiados para esta especie, por la forma característica en la que se disponen los nudos de forma verticilada y que influyen en la metodología de valoración, al considerarlos como nudos de margen y causar una excesiva penalización del material. Será necesario en estudios posteriores analizar la correspondencia de estos resultados con los de resistencia reales.

Tabla 3. Asignación total de clases resistentes segœn clases de calidad.

\begin{tabular}{|c|c|c|c|c|c|c|c|c|}
\hline \multirow[t]{2}{*}{$\begin{array}{c}\text { Clases de } \\
\text { calidad } \\
\text { visual }\end{array}$} & \multirow[t]{2}{*}{$\begin{array}{l}\mathbf{N}^{\circ} \text { piezas/ } \\
\text { Rendimiento }\end{array}$} & \multicolumn{2}{|c|}{$\begin{array}{c}\text { Datos de } \\
\text { resistencia } \\
\left(\mathrm{N} / \mathrm{mm}^{2}\right)\end{array}$} & \multicolumn{2}{|c|}{$\begin{array}{c}\text { Datos de } \\
\text { rigidez } \\
\left(\mathrm{N} / \mathrm{mm}^{2}\right)\end{array}$} & \multicolumn{2}{|c|}{$\begin{array}{c}\text { Datos de } \\
\text { densidad } \\
\left(\mathrm{kg} / \mathrm{m}^{3}\right)\end{array}$} & \multirow[t]{2}{*}{$\begin{array}{l}\text { Asignación } \\
\text { clase } \\
\text { resistente }\end{array}$} \\
\hline & & $f$ & $f_{05}$ & $\boldsymbol{E}_{m g}$ & $E_{m l}$ & $\rho_{m}$ & $\rho_{05}$ & \\
\hline ME1 & $161 / 21 \%$ & 50,7 & 29,6 & 10734 & 11264 & 504 & 426 & $\mathrm{C} 24$ \\
\hline ME2 & $320 / 42 \%$ & 39,8 & 18,0 & 10590 & 11077 & 484 & 400 & $\mathrm{C} 18$ \\
\hline Rechazos & $287 / 37 \%$ & 28,7 & 13,8 & 9063 & 9092 & 461 & 386 & - \\
\hline
\end{tabular}

Asimismo, se analizaron las relaciones entre las variables predictoras, mostrando los resultados en las Tablas 4 y 5 . Son de especial interés aquellas que para su obtención no impliquen dañar el material y logren una buena estimación de las propiedades resistentes. Por este motivo, se incorporaron variables como el módulo de elasticidad o la medida del tamaño de los nudos respecto a las dimensiones. Análogamente se añadió la variable densidad en los modelos ya que, aunque su estimación según normativa supone el deterioro del material, existen actualmente otras metodologías no destructivas para su evaluación, si bien todavía en fase de estudio, que podrían ser utilizadas en un futuro próximo.

Tabla 4. Modelos de regresión lineales para $f_{m}$ independiente.

\begin{tabular}{|l|c|c|c|c|c|}
\hline \multirow{2}{*}{ Relación } & \multicolumn{3}{c|}{ Coeficientes de la regresión } & \multicolumn{2}{c|}{$\begin{array}{c}\text { Coeficiente } \\
\text { determinación }\end{array}$} \\
\cline { 2 - 6 } & $\boldsymbol{A}$ & $\boldsymbol{B}$ & $\boldsymbol{C}$ & $\boldsymbol{D}$ & $\boldsymbol{R}^{2}$ \\
\hline$f_{m}=A+B x E_{m g}$ & $\begin{array}{c}-12,47 \\
* * *\end{array}$ & $\begin{array}{c}0,0053 \\
* * *\end{array}$ & - & - & 50,72 \\
\hline$f_{m}=A+B x \rho_{m}$ & $\begin{array}{c}-45,75 \\
* * *\end{array}$ & $\begin{array}{c}0,1736 \\
* * *\end{array}$ & - & - & 29,63 \\
\hline$f_{m}=A+B x D C A R E L$ & $\begin{array}{c}50,40 \\
* * *\end{array}$ & $\begin{array}{c}-37,15 \\
* * *\end{array}$ & - & - & 26,79 \\
\hline$f_{m}=A+B x D C T O R E L$ & $\begin{array}{c}44,81 \\
* * *\end{array}$ & $\begin{array}{c}-20,15 \\
* * *\end{array}$ & - & - & 20,07 \\
\hline$f_{m}=A+B x E_{m g}+C x h+D x t$ & $\begin{array}{c}-7,36 \\
* *\end{array}$ & $\begin{array}{c}0,0053 \\
* * *\end{array}$ & $\begin{array}{c}-0,035 \\
* *\end{array}$ & $\begin{array}{c}0,0030 \\
\mathrm{NS}\end{array}$ & 51,03 \\
\hline$f_{m}=A+B x \rho_{m}+C x h+D x t$ & $\begin{array}{c}-46,58 \\
* * *\end{array}$ & $\begin{array}{c}0,17 \\
* * *\end{array}$ & $\begin{array}{c}0,016 \\
\mathrm{NS}\end{array}$ & $\begin{array}{c}-0,029 \\
\mathrm{NS}\end{array}$ & 29,69 \\
\hline$f_{m}=A+B x E_{m g}+C x D C A R E L+D x D C T O R E L$ & $\begin{array}{c}10,66 \\
* * *\end{array}$ & $\begin{array}{c}0,0037 \\
* * *\end{array}$ & $\begin{array}{c}-18,16 \\
* * *\end{array}$ & $\begin{array}{c}-7,81 \\
* * *\end{array}$ & 54,30 \\
\hline$f_{m}=A+B x \rho_{m}+C x D C A R E L+D x D C T O R E L$ & $\begin{array}{c}14,29 \\
* *\end{array}$ & $\begin{array}{c}0,072 \\
* * *\end{array}$ & $\begin{array}{c}-23,77 \\
* * *\end{array}$ & $\begin{array}{c}-11,42 \\
* * * *\end{array}$ & 39,26 \\
\hline
\end{tabular}

***Significativo al $99 \%$. **Significativo al $95 \%$. *Significativo al $90 \%$. NS No significativo.

$h$ : anchura de cara de la pieza (mm).

$t$ : espesor de la pieza $(\mathrm{mm})$.

DCAREL: Diámetro relativo del nudo de cara respecto a la anchura de cara.

DCTOREL: Diámetro relativo del nudo de canto respecto al espesor. 
De la Tabla 4, se confirma que la mejor variable predictora directa de la resistencia de esta especie es el módulo de elasticidad (50,72\%). Estos resultados concuerdan con lo obtenido en el estudio de otras especies de España como el Pinus sylvestris L. (Hermoso, 2001) o el Pinus nigra Arn. (Conde, 2004). Pero además la calidad de la predicción puede ser ligeramente mejorada si se adiciona al modelo el valor de la anchura de cara $(51,03 \%)$.

Sin embargo, el espesor de la pieza resulta no significativo en los modelos en los que se ha considerado; no siendo así si lo que se introduce es el valor del diámetro relativo del nudo respecto al espesor de la viga (DCTOREL), en donde el grado de significación alcanza el $99 \%$, análogo a la variable del diámetro relativo del nudo respecto a la cara (DCAREL). Este resultado, por otro lado, refleja la influencia de los diámetros grandes en la disminución de la sección resistente residual de trabajo de la pieza. El añadir éstos parámetros relativos en las regresiones simples del módulo de elasticidad o la densidad, producen también mejoras sensibles $(54,30 \%$ y $39,26 \%$ respectivamente) y quedando patente en todos los casos su alta significación en el modelo (99\%).

Tabla 5. Modelos de regresión lineales para $E_{m g}$ independiente.

\begin{tabular}{|l|c|c|c|c|c|}
\hline \multirow{2}{*}{ Relación } & \multicolumn{3}{c|}{ Coeficientes de la regresión } & $\begin{array}{c}\text { Coeficiente } \\
\text { determinación }\end{array}$ \\
\cline { 2 - 6 } & $\boldsymbol{A}$ & $\boldsymbol{B}$ & $\boldsymbol{C}$ & $\boldsymbol{D}$ & $\boldsymbol{R}^{2}$ \\
\hline$E_{m}=A+B x \rho_{m}$ & $\begin{array}{c}-3587,99 \\
* * *\end{array}$ & $\begin{array}{c}27,32 \\
* * *\end{array}$ & - & - & 39,92 \\
\hline$E_{m}=A+B x D C A R E L$ & $\begin{array}{c}10896,6 \\
* * *\end{array}$ & $\begin{array}{c}-3700,99 \\
* * *\end{array}$ & - & - & 14,00 \\
\hline$E_{m}=A+B x D C T O R E L$ & $\begin{array}{c}10320,6 \\
* * *\end{array}$ & $\begin{array}{c}-1958,64 \\
* * *\end{array}$ & - & - & 10,77 \\
\hline$E_{m}=A+B x \rho_{m}+C x h+D x t$ & $\begin{array}{c}-4422,36 \\
* * *\end{array}$ & $\begin{array}{c}27,44 \\
* * *\end{array}$ & $\begin{array}{c}8,96 \\
* * *\end{array}$ & $\begin{array}{c}-11,71 \\
*\end{array}$ & 40,94 \\
\hline$E_{m}=A+B x \rho_{m}+C x D C A R E L+D x D C T O R E L$ & $\begin{array}{c}-226,02 \\
\text { NS }\end{array}$ & $\begin{array}{c}21,73 \\
* * *\end{array}$ & $\begin{array}{c}-1185,70 \\
* * *\end{array}$ & $\begin{array}{c}-998,7 \\
* * *\end{array}$ & 41,50 \\
\hline
\end{tabular}

$* * *$ Significativo al 99\%. $* *$ Significativo al $95 \%$. $*$ Significativo al $90 \%$. NS No significativo.

Otra variable necesaria para el cálculo estructural es el módulo de elasticidad; por este motivo también se consideró en este estudio el análisis de las variables que pueden intervenir para su estimación, teniendo presente que puedan ser obtenidas de forma sencilla y sin dañar al material para un uso posterior.

Los resultados del análisis de los predictores del módulo de elasticidad (Tabla 5), muestran que la mejor estima se tiene a través de la densidad, además de la resistencia como se vio en la Tabla 4, siendo nuevamente mejorada notablemente por la inclusión de los parámetros relativos a la dimensión de la pieza. En esta ocasión, aunque en menor medida que las otras variables, el espesor es significativo al $90 \%$ en el modelo.

Las variables relativas que consideran la influencia de los nudos en la sección, por sí solas, no son buenas estimadoras del módulo de elasticidad como se extrae de los resultados de los coeficientes de determinación, aunque sí son significativas en el modelo lineal. De nuevo, si se consideran como complemento a otras variables generando modelos múltiples de predicción, no sólo son significativas sino que mejoran la calidad de la estimación sensiblemente. 


\section{CONCLUSIONES}

- De la comparación de las propiedades mecánicas obtenidas con las ya existentes de estudios anteriores, se deriva que la calidad de la madera no ha variado tras la aplicación de tratamientos selvícolas orientados a un crecimiento rápido.

- Se puede confirmar en el pino radiata del País Vasco el cumplimiento de las clases resistentes que actualmente aparecen en la normativa UNE-EN 1912, que establecen para la primera calidad visual C24 y para la segunda C18.

- Es necesario realizar estudios encaminados a la valoración de la norma actual de clasificación visual española (UNE 56.544) para las peculiaridades de la madera de pino radiata.

- Se confirma como mejor predictor simple de la resistencia el módulo de elasticidad para el pino radiata, si bien puede ser mejorado introduciendo las variables relativas a la dimensión de la pieza.

- La densidad es un buen estimador de la rigidez de la madera de pino radiata, no teniendo trascendencia las predicciones simples utilizando parámetros referentes a la anchura de cara o el espesor. La consideración de modelos de predicción múltiples con parámetros relativos al tamaño unido a la densidad, enriquecen notablemente la calidad de las predicciones.

\section{AGRADECIMIENTOS}

Este trabajo se enmarca dentro del Proyecto AGL2004-01598/FOR del Programa Nacional de I+D sobre Recursos y Tecnologías Agroalimentarias. Se agradece especialmente la colaboración en los trabajos de ensayo al Dr. Rafael Díez Barra así como al equipo de trabajo del Laboratorio de Estructuras de Madera del CIFOR-INIA.

\section{REFERENCIAS}

Ceballos, L.; Ruiz de la torre, J. 1979. Árboles y Arbustos de la España Penínsular. IFIE, España.

Conde, M. 2004. Caracterización de la madera estructural de Pinus nigra Subsp. Salzmannii”. Tesis Doctoral, Universidad Politécnica de Madrid, España.

Fernández-Golfín seco, J.I.; Díez Barra, M.R.; Gutiérrez Oliva, A. 1997. Caracterización mecánica de la madera aserrada de pino silvestre de los sistemas Central e Ibérico mediante probetas de tamaño estructural. Revista de Investigación Agraria 6 (1 y 2): 183-215.

Fernández-Golfín, J.I.; Díez, M.R. 1999. Coníferas españolas de uso estructural. Propiedades de la madera aserrada. Revista AITIM 200: 72-75.

Fernández-Golfín Seco, J.I.; Díez Barra, M.R., Conde García, M.; Hermoso Prieto, E. 2000. Caracterización de la madera aserrada con destino estructural efectuada de acuerdo con las normas europeas: aplicación al caso del pino laricio español. I Congreso Iberoamericano de Investigación y Desarrollo de Productos Forestales, Universidad de Concepción (Chile).

Fernandez-Golfin, J.I.; Gutiérrez, A.; Baonza, M.V.; Díez, M.R.; Alvárez, H.; Rodríguez, E.; Troya, M.T.; Conde, M. ; Hermoso, E. 2001 a. Caracterización de la madera de Pinus nigra subsp. salzmannii. Final report of project SC96-045-C2-2. Informe interno. 
Fernández-Golfín, J.I.; Díez, M.R.; Hermoso, E. 2001 b. Propiedades mecánicas de la madera de pino laricio obtenidas con probetas de tamaño estructural. Actas III Congreso Forestal Español, Granada: 567-572.

Fernández-Golfín Seco, J.I.; Díez Barra, M.R.; Baonza MerinO, M.V.; Gutiérrez Oliva, A.; Prieto Hermoso, E.; Conde García, M.; Van den Eynde, V. 2001c. Caracterización de la calidad y las propiedades de la madera de pino laricio (Pinus nigra). Revista de Investigación Agraria 10 (2): 311-332.

Hermoso, E.; Fernández-Golfín J.I.; Díez M.R. 2002. Análisis del efecto del volumen sobre la resistencia característica a flexión de la madera de los pinos silvestre y laricio de procedencia española. Materiales de Construcción 268 (52): 43-55.

Fernández-Golfín J.I.; Díez Barra, M.R.; Hermoso Prieto, E. 2003. Relationships between grade determining properties of Spanish Scots and laricio pine structural timber. Materiales de Construcción 53 (270): 45-55.

Hermoso Prieto, E. 2001. Caracterización mecánica de la madera estructural de Pinus sylvestris L. Tesis Doctoral, Universidad Politécnica de Madrid, España.

Hermoso, E.; Fernández-Golfín J.I.; Díez M.R. 2002. Madera estructural de pino silvestre: Caracterización Mecánica. Revista de Investigación Agraria 11 (2): 425-440.

Hermoso, E.; Fernández-Golfín J.I.; Díez M.R. 2003. Mechanical characterization of timber according to European standards from Spanish provenances of Scots Pine. Revista de Investigación Agraria 12 (3): 103-110.

Ministerio de Agricultura Pesca y Alimentación. 1991. Propiedades y Tecnología de la madera de pino radiata del País Vasco. Monografías INIA n ${ }^{\circ} 80$.

Ortiz, J.; Cruz, H.; Blanchon, J.L. 1990. Informe final del proyecto MA1B-0129 Standard Quality of Pinus pinaster. Informe interno.

Ortiz, J.; Martínez, J.J. 1991. Características mecánicas de la madera de pino gallego, obtenidas a partir de ensayos con piezas de tamaño estructural. Revista AITIM 150: 95-101.

UNE 56544: 2003. Clasificación visual de la madera aserrada para uso estructural. AENOR, España.

UNE-EN 338: 2003. Madera estructural. Clases resistentes. AENOR, España.

UNE-EN 384: 2004. Madera estructural. Determinación de los valores característicos de las propiedades mecánicas y la densidad. AENOR, España.

UNE-EN 408: 2004. Madera estructural. Madera estructural y madera laminada. Determinación de algunas propiedades físicas y mecanicas. AENOR, España.

UNE-EN 1912: 2004. Madera estructural. Clases resistentes. Asignación de especies y calidades visuales. Madera de Coníferas. AENOR, España. 